\title{
Can we achieve complete remission in locally advanced unresectable Hepatocellular Carcinoma (HCC) by using Sorafenib? Case report and review of literature from Qatar
}

\author{
Kakil Ibrahim Rasul \\ National Center for Cancer Care and Research (NCCCR), Doha, Qatar; kakil954@yahoo.com
}

Received 7 May 2012; revised 20 June 2012; accepted 18 July 2012

\begin{abstract}
Patients with HCC continue to have a dismal prognosis, with 1-year and 3-year survival rates of 36 and $17 \%$, respectively. This is in part related to more than two-thirds of tumors being diagnosed at advanced stages, as well as a substantial portion of patients with early HCC failing to receive potentially curative treatments. Systemic therapy for advanced unresectable HCC limited until the discovery of sorafenib, we are reporting a 53 year patient with unresectable HCC achieved complete remission clinically, radiologically and biochemically with sorafenib which is the first case report.
\end{abstract}

Keywords: Hepatocellular Ca; Sorafenib; Chemoembolization

\section{INTRODUCTION}

Patients with HCC continue to have a dismal prognosis, with 1-year and 3-year survival rates of 36 and 17\%, respectively [1]. Sorafenib (Nexavar Payer-Schering) is the only systemic therapy indicated to treat $\mathrm{HCC}$, in two Phase III studies (SHARP and Asia-Pacific). Sorafenib significantly improved OS in patients with unresectable HCC (uHCC) [2,3]. There is no report of complete remission in all the studies used sorafenib in unresectable HCC.

The efficacy of sorafenib has been recently investigated in a phase III trial, reported in abstract form at the 2007 American Society of Clinical Oncology meeting. LLovet and colleagues randomized 602 patients with Child Class A cirrhosis and hepatocellular carcinoma to sorafenib versus placebo group [4]. The overall results were encouraging. Treatment with sorafenib significantly improved survival (hazard ratio for all cause mortality: $0.69, P=0.0006)$. Treatment was also associated with an increased time to disease progression (5.5 mo vs $2.8 \mathrm{mo}$ ) and disease control rate ( $43 \%$ versus $32 \%$ ). But there is no complete remission in these studies. Overall toxicity did not differ between treatment and placebo arm (52\% versus $54 \%$ ). Based on this trial, sorafenib has become the most promising chemotherapeutic agent in the treatment of hepatocellular carcinoma in patients with preserved liver function.

\section{CASE PRESENTATION}

A 53 years male Egyptian known non insulin dependent diabetes mellitus (NIDDM) on Metformin $500 \mathrm{mg}$ orally twice daily since 6 years, and $\mathrm{HCV}+$ ve Chronic smokerlpack/day since 35 years On December 2010 during follow up ultrasound examination found to have focal lesion in the liver He had mild right hypochondrial pain which dull in nature not radiating and no associated symptoms, his ECOG (Eastern Cooperative Oncology Group performance status) PS is 0, Child-pugh assessment of the liver scored A. laboratory tests, AFP 13,241 I.U/L, normal kidney function, complete blood picture and PT, APPT. after nearly 4 weeks AFP level raised to 21,227 I.U/L, CT Scan showed cirrhotic liver with large lesion $(6 \times 7 \mathrm{~cm})$ in the right lobe of the liver with typical radiological features of HCC (arterial enhancement and venous wash out) (Figure 1). He had first session of Tans-arterial chemo-embolization on mid Feb 2011, CT scan repeated after 2 weeks showed marked enlargement of the lesion become involving nearly whole right lobe of the liver with thrombosis of the right branch of the portal vein (Figure 1), AFP level raised to 48,928 I.U/L, so further local treatment was stopped and patient started on Sorafenib $400 \mathrm{mg}$ orally twice daily on 7 March 2011. By June 2011 his AFP was back to normal and his CT scan showed dramatic decrease in size of the tumor and maintained normal AFP (Figure 2) till now and his last CT scan on Jan, 2012 show nearly complete remission of the lesion and recanalization of the left main portal vein 

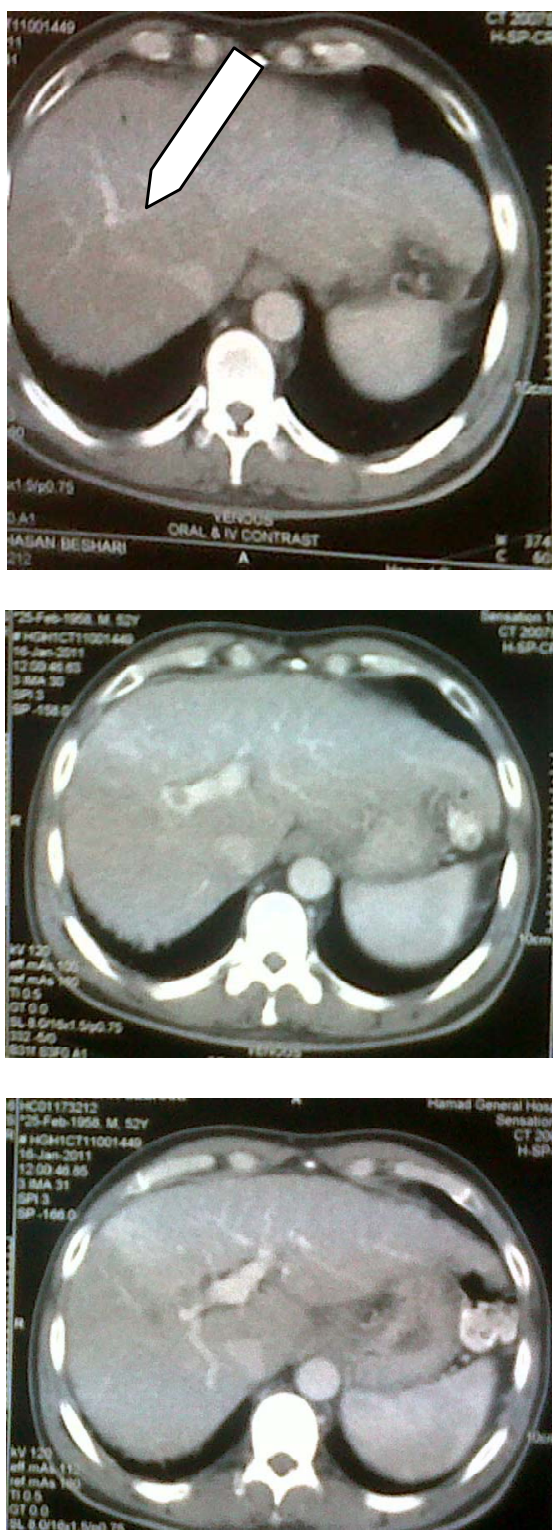

Figure 1. CT scan Jan 2011 showed big lesion in the right lobe of the liver.

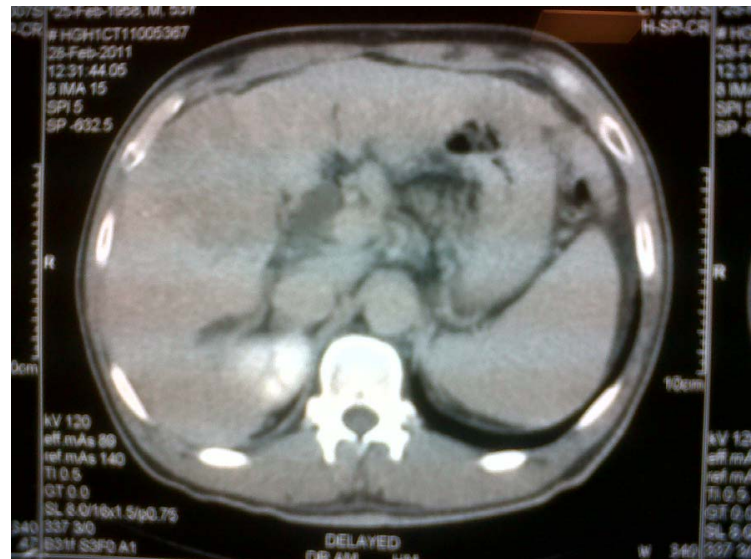

Figure 2. After chemoemb \& before sorafennib.

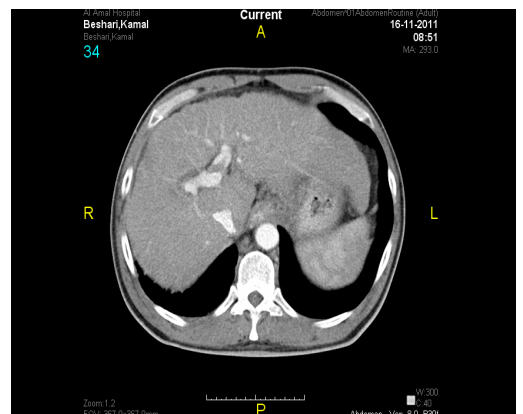

Figure 3. After sorafeninb.

(Figure 3). He developed the usual side effects which are reported from the previous studies of sorafenib, skin rash, hand foot syndrome (Figure 4), hypertension and fatigue.

\section{DISCUSSION}

Patients with hepatocellular carcinoma (HCC) have a poor prognosis, with a 5-year survival rate of less than $5 \%$ and a median survival rate of less than 4 months if the disease is unresectable [5-7]. Indeed, curative treatment (liver transplantation, surgical resection, percutaneous ablation) can only be performed in less than $25 \%$ of patients. This is either because of contraindications to surgery (advanced cirrhosis in particular), the presence of locally advanced disease (multifocal lesions, invasion of the portal vein), or, more rarely, technical reasons (lesion difficult to access, subcapsular or diaphragmatic location). For locally advanced unresectable HCC until recently we have limited option for treatment of such cases, systemic chemotherapy either as single agent or in combination were not helpful. We introduction of sorafenib lead to hope for such patients. In the phase 3 Sorafenib HCC Assessment Randomized Protocol (SHARP) trial that randomized patients with advanced HCC to sorafenib versus placebo patients with Child-Pugh A were allowed to be on the study as were patients with microscopic vascular disease or extrahepatic disease and an Eastern Cooperative Oncology Group (ECOG) performance status of 0 to 2 . Patients were allowed to be randomized to sorafenib or placebo and to stay in the study until radiologic progression and also clinical progression. This study showed an improvement in survival for the sorafenib arm of 10.7 months versus 7.9 months with placebo with a clinical significance and statistical significance depicted by hazard ratio of 0.69 and a $P$ value of 0.00058 [1]. Another study Asian pacific study Improvement in OS for the patients randomized to sorafenib in the Asia-Pacific Study was consistent with the SHARP ${ }^{\dagger}$ Trial as indicated by hazard ratios for survival (HR: 0.68 and 0.69, respectively), Like the SHARP Trial, the AsiaPacific Study was stopped early and patients taking placebo crossed over to receive Nexavar [1,2]. We are reporting a case with $\mathrm{HCV}$ who developed $\mathrm{HCC}$ which was 
Test Selected: AFP - A-Feto Protein FROM: 02/11/2011 12:22 TO: 05/01/2011 08:07

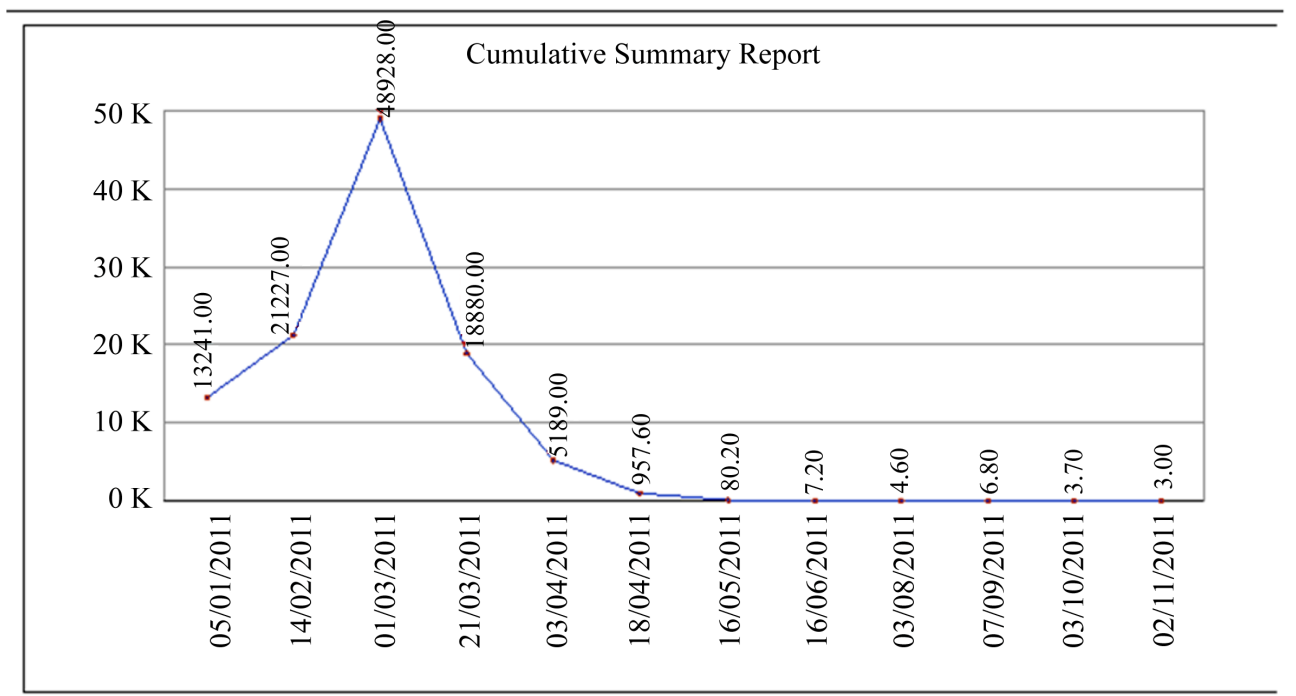

Figure 4. AFP level during treatment, Nov. 2011-Jan. 2012.
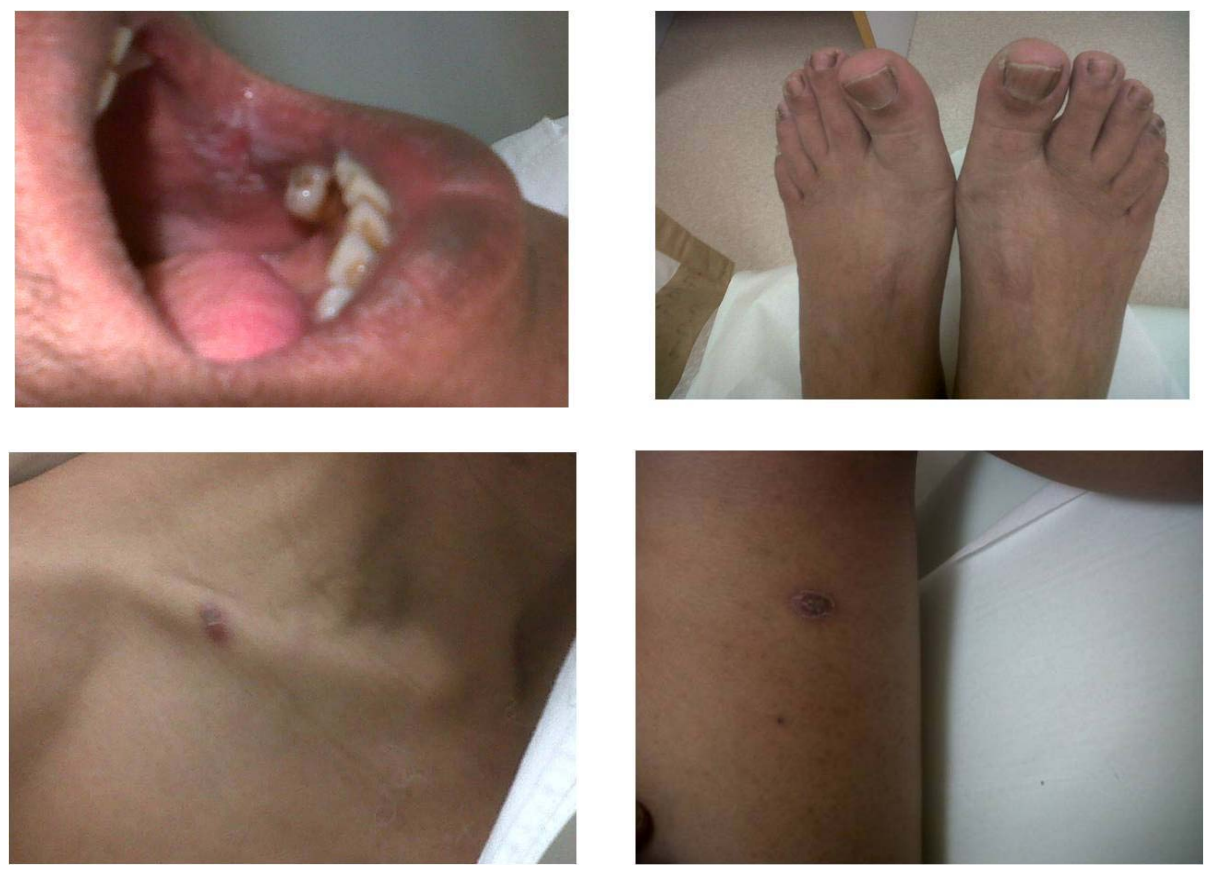

Figure 5. Side effects of sorafenib mouth ulcers, hand foot syndrome and skin rash.

rapidly progressing over 2 months and did not benefit from local treatment (TACE) who responded dramatically to sorafeinib and maintained this response near complete remission for more than 6 months now. It $1^{\text {st }}$ case of complete remission, none of the previous study reported complete remission with sorafenib treatment. He developed the known side effects of sorafenib as skin rash, hypertension and fatigue (Figure 5).

\section{CONCLUSION}

This is the first case of $\mathrm{HCV}$ associated HCC reporting complete remission with sorafenib.

\section{REFERENCES}

[1] El-Serag, H.B., Siegel, A.B., Davila, J.A., et al. (2006) Treatment and outcomes of treating of hepatocellular carcinoma among Medicare recipients in the United States: A population-based study. Journal of Hepatology, 44, 158166. doi:10.1016/j.jhep.2005.10.002

[2] Josep, M. Llovet, M.D., Ricci, S., Mazzaferro, V., Hilgard, P., Gane, E., et al. (2008) Sorafenib in advanced hepatocellular carcinoma. The New England Journal of Medi- 
cine, 359, 378-390. doi:10.1056/NEJMoa0708857

[3] Cheng, A.L., Kang, Y.K., Chen, Z., Tsao, C.J., et al. (2009) Efficacy and safety of sorafenib in patients in the AsiaPacific region with advanced hepatocellular carcinoma: A phase III randomised, double-blind, placebo-controlled trial. The Lancet Oncology, 10, 25-34. doi:10.1016/S1470-2045(08)70285-7

[4] Forner, A., Hessheimer, A.J., Isabel Real, M., et al. (2006) Treatment of hepatocellular carcinoma. Critical Reviews in Oncology/Hematology, 60, 89-98.

doi:10.1016/j.critrevonc.2006.06.001
[5] Okuda, K. (2000) Hepatocellular carcinoma. Journal of Hepatology, 32, 225-237. doi:10.1016/S0168-8278(00)80428-6

[6] Llovet, J.M., Bustamante, J., Castells, A., et al. (1999) Natural history of unresected nonsurgical hepatocellular carcinoma for the design and evaluation of therapeutic trials. Hepatology, 29, 62-67. doi:10.1002/hep.510290145

[7] Okuda, K., Takayasu, K., Okada, S. (1997) Treatment selection. In: Okuda, K. and Tabor, E. Eds., Liver Cancer, Churchill-Livingstone, New York, 435-439. 